\title{
The Shi arrangement and the Ish arrangement
}

\author{
Drew Armstrong ${ }^{1}$ and Brendon Rhoades ${ }^{2}$ \\ ${ }^{1}$ Department of Mathematics, University of Miami, Coral Gables, FL, USA \\ ${ }^{2}$ Department of Mathematics, MIT, Cambridge, MA, USA
}

\begin{abstract}
This paper is about two arrangements of hyperplanes. The first — the Shi arrangement — was introduced by Jian-Yi Shi to describe the Kazhdan-Lusztig cells in the affine Weyl group of type $A$. The second - the Ish arrangement - was recently defined by the first author who used the two arrangements together to give a new interpretation of the $q, t$-Catalan numbers of Garsia and Haiman. In the present paper we will define a mysterious "combinatorial symmetry" between the two arrangements and show that this symmetry preserves a great deal of information. For example, the Shi and Ish arrangements share the same characteristic polynomial, the same numbers of regions, bounded regions, dominant regions, regions with $c$ "ceilings" and $d$ "degrees of freedom", etc. Moreover, all of these results hold in the greater generality of "deleted" Shi and Ish arrangements corresponding to an arbitrary subgraph of the complete graph. Our proofs are based on nice combinatorial labellings of Shi and Ish regions and a new set partition-valued statistic on these regions.

Résumé. Cet article traite de deux arrangements d'hyperplans. Le premier — arrangement Shi — a été introduit par Jian-Yi Shi pour décrire les cellules de Kazhdan-Lusztig du groupe de Weyl affine de type A. Le deuxième - arrangement Ish - a été récemment défini par le premier auteur pour donner une nouvelle interprétation des nombres $q, t$-Catalan de Garsia et Haiman. Ici nous définissons une mystérieuse "symétrie combinatoire" entre les deux arrangements et nous montrons que cette symétrie conserve un grand nombre d'informations. Par exemple, les arrangements Shi et Ish ont le même polynôme caractéristique, le même nombre de régions, de régions bornées, de régions dominantes, de régions avec $c$ "plafonds" et $d$ "degrés de liberté", etc. En outre, ces résultats se généralisent aux arrangements Shi et Ish "deleted" correspondant à un sous-graphe arbitraire du graphe complet. Nos preuves reposent sur des étiquetages combinatoires des régions Shi et Ish, et sur une nouvelle statistique associée.
\end{abstract}

Keywords: hyperplane arrangement, nonnesting partition, product formula

\section{Introduction}

A hyperplane arrangement is a finite collection of affine hyperplanes in Euclidean space. Some of the nicest arrangements come from the reflecting hyperplanes of Coxeter groups. In particular, the Coxeter arrangement of type $A$ (also known as the braid arrangement) is the arrangement in $\mathbb{R}^{n}$ defined by

$$
\operatorname{Cox}(n):=\left\{x_{i}-x_{j}=0: 1 \leq i<j \leq n\right\} .
$$

Here $\left\{x_{1}, \ldots, x_{n}\right\}$ are the standard coordinate functions on $\mathbb{R}^{n}$. 

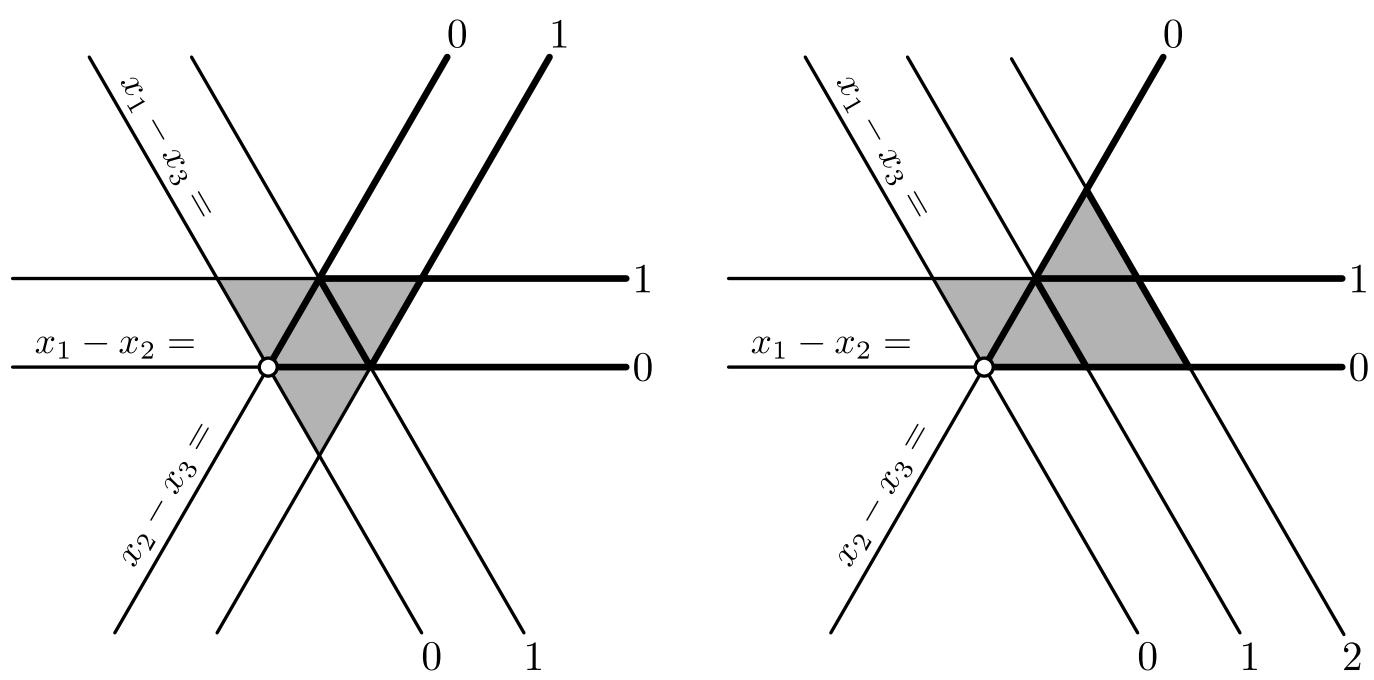

Fig. 1: The arrangements Shi(3) (left) and Ish(3) (right)

Postnikov and Stanley [9] introduced the idea of a deformation of the Coxeter arrangement - this is an affine arrangement each of whose hyperplanes is parallel to some hyperplane of the Coxeter arrangement. In the present paper we will study two specific deformations of the Coxeter arrangement and we will observe a deep similarity between them. The first is the Shi arrangement which was one of Postnikov and Stanley's motivating examples:

$$
\operatorname{Shi}(n):=\operatorname{Cox}(n) \cup\left\{x_{i}-x_{j}=1: 1 \leq i<j \leq n\right\} .
$$

This arrangement was defined by Jian-Yi Shi [11, Chapter 7] to study of the Kazhdan-Lusztig cellular structure of the affine Weyl group of type A. The second is the Ish arrangement, recently defined by the first author [1]:

$$
\operatorname{Ish}(n):=\operatorname{Cox}(n) \cup\left\{x_{1}-x_{j}=i: 1 \leq i<j \leq n\right\} .
$$

He used the Shi and Ish arrangements to give a new description of the $q, t$-Catalan numbers of Garsia and Haiman in terms of the affine Weyl group of type $A$. Figure 1 displays the arrangements $\operatorname{Shi}(3)$ and $\operatorname{Ish}(3)$. (Note that the normals to the hyperplanes of either $\operatorname{Shi}(n)$ or $\operatorname{Ish}(n) \operatorname{span}$ the hyperplane $x_{1}+x_{2}+\cdots+x_{n}=0$. Hence we will always draw their restrictions to this space.)

The heart of this paper is the following correspondence between Shi and Ish hyperplanes. The correspondence is natural to state but we find it geometrically mysterious. We will call this a "combinatorial symmetry":

$$
x_{i}-x_{j}=1 \longleftrightarrow x_{1}-x_{j}=i \text { for } 1 \leq i<j \leq n
$$

This symmetry allows us to define deleted versions of the Shi and Ish arrangements. Let $\left(\begin{array}{c}{[n]} \\ 2\end{array}\right)$ denote the set of pairs $i j$ satisfying $1 \leq i<j \leq n$ and consider a simple loopless graph $G \subseteq\left(\begin{array}{c}{[n]} \\ 2\end{array}\right)$. The deleted Shi and Ish arrangements are defined as follows:

$$
\operatorname{Shi}(G):=\operatorname{Cox}(n) \cup\left\{x_{i}-x_{j}=1: i j \in G\right\},
$$



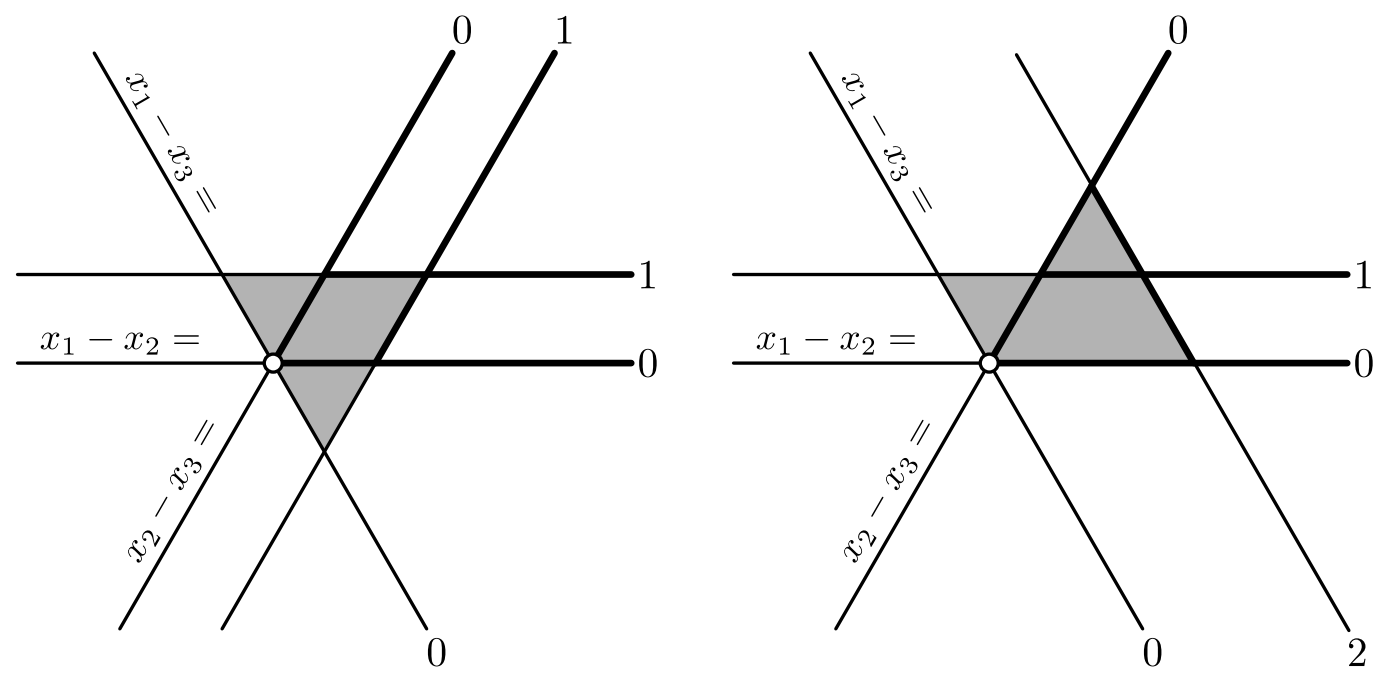

Fig. 2: The arrangements $\operatorname{Shi}(G)$ (left) and $\operatorname{Ish}(G)$ (right) corresponding to the "chain" $G=\{12,23\} \subseteq\left(\begin{array}{c}{[3]} \\ 2\end{array}\right)$

$$
\operatorname{Ish}(G):=\operatorname{Cox}(n) \cup\left\{x_{1}-x_{j}=i: i j \in G\right\} .
$$

The arrangement $\operatorname{Shi}(G)$ was first considered by Athanasiadis [3]. Note that $\operatorname{Shi}(G)(\operatorname{resp} . \operatorname{Ish}(G))$ interpolates between the Coxeter arrangement and the Shi (resp. Ish) arrangement. That is, if $\emptyset \in\left(\begin{array}{c}{[n]} \\ 2\end{array}\right)$ is the "empty" graph and $K_{n}=\left(\begin{array}{c}{[n]} \\ 2\end{array}\right)$ is the "complete" graph, we have that $\operatorname{Shi}(\emptyset)=\operatorname{Ish}(\emptyset)=\operatorname{Cox}(n)$, $\operatorname{Shi}\left(K_{n}\right)=\operatorname{Shi}(n)$, and $\operatorname{Ish}\left(K_{n}\right)=\operatorname{Ish}(n)$. Figure 2 displays the arrangements $\operatorname{Shi}(G)$ and $\operatorname{Ish}(G)$ corresponding to the "chain" $G=\{12,23\} \subseteq\left(\begin{array}{c}{[3]} \\ 2\end{array}\right)$.

To state our Main Theorem right away, we need a few definitions. Let $\mathcal{A}$ be a hyperplane arrangement in $\mathbb{R}^{n}$. The intersection poset $\mathfrak{L}(\mathcal{A})$ of $\mathcal{A}$ is the collection of all nonempty intersections of the hyperplanes in $\mathcal{A}$ partially ordered by reverse inclusion. The poset $\mathfrak{L}(\mathcal{A})$ has the structure of a graded meet-semilattice [13] with unique minimal element given by the 'empty intersection' $\mathbb{R}^{n}$. Let $\mu: \mathfrak{L}(\mathcal{A}) \times \mathfrak{L}(\mathcal{A}) \rightarrow \mathbb{Z}$ be the Möbius function of $\mathfrak{L}(\mathcal{A})$ (see [12]). The characteristic polynomial $\chi_{\mathcal{A}}(p) \in \mathbb{Z}[p]$ of $\mathcal{A}$ is the polynomial

$$
\chi_{\mathcal{A}}(p):=\sum_{X \in \mathfrak{L}(\mathcal{A})} \mu\left(\mathbb{R}^{n}, X\right) p^{\operatorname{dim}(X)}
$$

The characteristic polynomial of $\mathcal{A}$ determines the Hilbert series of the Orlik-Solomon algebra of $\mathcal{A}$ and the Hilbert series of the cohomology ring of the complement of the complexification $\mathcal{A}_{\mathbb{C}}:=\left\{\mathbb{C} \otimes_{\mathbb{R}} H\right.$ : $H \in \mathcal{A}\}$ of $\mathcal{A}$ in $\mathbb{C}^{n}$ (where cohomology is computed with coefficients in $\mathbb{C}$ ) [8].

If $\mathcal{A}$ is a hyperplane arrangement in $\mathbb{R}^{n}$, the connected components of the complement $\mathbb{R}^{n} \backslash \bigcup_{H \in \mathcal{A}} H$ are called the regions of $\mathcal{A}$. If $R$ is a region of $\mathcal{A}$, the recession cone of $R$ is

$$
\operatorname{Rec}(R):=\left\{v \in \mathbb{R}^{n}: R+v \subseteq R\right\}
$$

Since $R$ is convex, $\operatorname{Rec}(R)$ is closed under nonnegative linear combinations. The dimension of the cone $\operatorname{Rec}(R)$ is called the number of degrees of freedom of the region $R$. The region $R$ is bounded if and only if it has zero degrees of freedom. 
Let $R$ be a region of the arrangement $\mathcal{A}$ in $\mathbb{R}^{n}$. The hyperplanes in $\mathcal{A}$ decompose the topological closure $\bar{R}$ into faces of various dimensions. A hyperplane $H \in \mathcal{A}$ is called a wall of $R$ if $H$ is the affine span of a codimension-one face of $R$. A wall $H$ of $R$ is called a ceiling of $R$ if $H$ is not a linear hyperplane and if $H$ does not separate $R$ from the origin.

Main Theorem. Let $G \subseteq\left(\begin{array}{c}{[n]} \\ 2\end{array}\right)$ be a graph on $n$ vertices and let $c$ and $d$ be nonnegative integers. The hyperplane arrangements $\operatorname{Shi}(G)$ and $\operatorname{Ish}(G)$ have the following objects in common:

- the characteristic polynomial;

- the number of regions with c ceilings and d degrees of freedom;

- the number of dominant regions with c ceilings.

Proof. These are Theorems 3.1, 5.2, and 5.3, respectively.

For example, here are the joint distributions of ceilings $(c)$ and degrees of freedom $(d)$ for the arrangements in Figures 1 and 2 , respectively.

\begin{tabular}{|c|c|c|c|c|}
\hline & \multicolumn{3}{|c|}{$d$} \\
\hline & & 1 & 2 & 3 \\
\hline & 0 & & & 6 \\
\hline$c$ & 1 & 3 & 6 & \\
\hline & 2 & 1 & & \\
\hline
\end{tabular}

\begin{tabular}{|c|c|c|c|c|}
\hline & \multicolumn{3}{|c|}{$d$} \\
\hline & & 1 & 2 & 3 \\
\hline & 0 & & & 6 \\
\hline$c$ & 1 & 2 & 4 & \\
\hline & 2 & 1 & & \\
\hline
\end{tabular}

We find it mysterious that the correspondence $x_{i}-x_{j}=1 \leftrightarrow x_{1}-x_{j}=i$ preserves so much information. However, it does not preserve everything. It can be shown using Figure 1 that $\operatorname{Shi}(G)$ and $\operatorname{Ish}(G)$ do not have the same intersection poset in general. It can also be shown that $\operatorname{Shi}(G)$ and $\operatorname{Ish}(G)$ do not have the same Tutte polynomial in general and that the Orlik-Solomon algebras of these arrangements are not in general graded-isomorphic (although by our Main Theorem these algebras do have the same Hilbert series). The authors are interested in a more compact statement of the Main Theorem as well as a more conceptual understanding of what is preserved and what is not preserved by the Shi/Ish duality.

The remainder of the paper is structured as follows. In Section 2 we review and introduce some notation related to set partitions. In Section 3 we prove that the arrangements $\operatorname{Shi}(G)$ and $\operatorname{Ish}(G)$ have the same characteristic polynomial and give an explicit expression for this polynomial. This expression is an alternating sum involving $G$-analogs of the Stirling numbers; it appears to be new even for the deleted Shi arrangements. In Section 4 we introduce a nice combinatorial labeling of the regions of $\operatorname{Shi}(G)$ and Ish $(G)$ and explain how this labeling can be used to read off the number of ceilings and number of degrees of freedom of a region. In Section $\mathbf{5}$ we introduce a new set partition-valued statistic called "ceiling partition" on the regions of $\operatorname{Shi}(G)$ and $\operatorname{Ish}(G)$ and show that the ceiling partition refines the "number of ceilings' statistic. We use the ceiling partitions to prove refined versions of Parts 2 and 3 of the Main Theorem.

\section{Background on Set Partitions}

If $\pi$ is a set partition of $[n]$, the arc diagram of $\pi$ is the graph on the vertex set $[n]$ whose edges are exactly the pairs $i j$ with $i<j$ such that $i$ and $j$ are blockmates in $\pi$ and there does not exist $k$ with $i<k<j$ such that $i, j$, and $k$ are blockmates in $\pi$. Figure 3 shows the arc diagram 


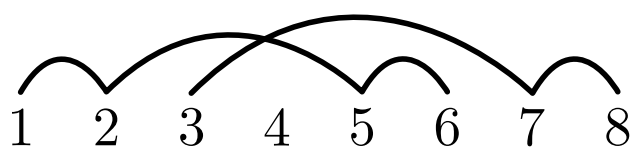

Fig. 3: A partition of [8] with type $(1,0,1,1,0,0,0,0)$

of the partition $\{\{1,2,5,6\},\{3,7,8\},\{4\}\}$ of [8]. The type of a partition $\pi$ of $[n]$ is the sequence $\left(r_{1}, \ldots, r_{n}\right) \in \mathbb{N}^{n}$, where $r_{i}$ is the number of blocks of $\pi$ of size $i$. The type of the partition of [8] in Figure 3 is $(1,0,1,1,0,0,0,0)$.

For $1 \leq k \leq n$, recall that the Stirling number (of the second kind) $\operatorname{Stir}(n, k)$ counts the number of partitions of $[n]$ with exactly $k$ blocks. In order to study the deleted Shi and Ish arrangements we will find it convenient to introduce a ' $G$-analog' of the Stirling numbers. For a graph $G \subseteq\left(\begin{array}{c}{[n]} \\ 2\end{array}\right)$, call a partition $\pi$ of $[n] G$-deleted if every edge in the arc diagram of $\pi$ is also an edge in $G$. Denote by $\operatorname{Stir}(G, k)$ the number of $G$-deleted partitions of $[n]$ with exactly $k$ blocks. For example, if $G=K_{n}$ is the complete graph we have that $\operatorname{Stir}\left(K_{n}, k\right)=\operatorname{Stir}(n, k)$ and if $G=\emptyset$ is the graph with no edges we have $\operatorname{Stir}(\emptyset, k)=\delta_{k, n}$.

A partition $\pi$ of $[n]$ is called nonnesting if there do not exist numbers $a<b<c<d$ such that both $a d$ and $b c$ are arcs in the arc diagram of $\pi$. The partition in Figure 3 is not nonnesting because the arcs 37 and 56 nest. The partition $\{\{1,3\},\{2,4\}\}$ of $[4]$ is nonnesting.

For $1 \leq d \leq n$, a partition $\pi$ of $[n]$ is said to have $d$ connected components if $d-1$ is the maximal length of a sequence $1<i_{1}<i_{2}<\ldots<i_{d-1} \leq n$ such that $\pi$ refines the set partition

$$
\left\{\left\{1,2, \ldots, i_{1}\right\},\left\{i_{1}+1, i_{1}+2, \ldots, i_{2}\right\}, \ldots,\left\{i_{d-1}, i_{d-1}+1, \ldots, n\right\}\right\} .
$$

(Some authors refer to connected components as "blocks" - we reserve this term for the elements of a set partition.)

\section{Characteristic Polynomials}

Our first result is that the characteristic polynomials of $\operatorname{Shi}(G)$ and $\operatorname{Ish}(G)$ are equal for any graph $G \subseteq\left(\begin{array}{c}{[n]} \\ 2\end{array}\right)$. We give an explicit formula for this common characteristic polynomial as an alternating sum involving the $G$-deleted Stirling numbers $\operatorname{Stir}(G, k)$.

Theorem 3.1. Let $G \subseteq\left(\begin{array}{c}{[n]} \\ 2\end{array}\right)$ be a graph on $n$ vertices. The characteristic polynomials of the deleted Shi and Ish arrangement are given by:

$$
\chi_{\operatorname{Shi}(G)}(p)=\chi_{\operatorname{Ish}(G)}(p)=p \sum_{k=0}^{n-1}(-1)^{k} \operatorname{Stir}(G, n-k)(p-k-1)(p-k-2) \cdots(p-n+1) .
$$

Proof. (Sketch.) Since the hyperplanes in $\operatorname{Shi}(G)$ and $\operatorname{Ish}(G)$ have defining equations over $\mathbb{Z}$, we can apply the finite fields method of Crapo and Rota [5] to compute these characteristic polynomials. Given a sufficiently large prime $p$, this amounts to showing that the number of points in the complements in $\mathbb{F}_{p}^{n}$ of the reductions of $\operatorname{Shi}(G)$ and $\operatorname{Ish}(G)$ modulo $p$ are both counted by the alternating sum in Equation 10 (here $\mathbb{F}_{p}$ denotes the field with $p$ elements). We use the Principle of Inclusion-Exclusion [12, Chapter 2] to perform this enumeration. 
Headley [6] showed that the characteristic polynomial of the 'full' Shi arrangement $\operatorname{Shi}(n)$ is given by $\chi_{\operatorname{Shi}(n)}(p)=p(p-n)^{n-1}$. Athanasiadis [4] later showed that when the graph $G$ avoids certain induced subgraphs, the arrangement $\operatorname{Shi}(G)$ is (inductively) free and the characteristic polynomial $\chi_{\operatorname{Shi}(G)}(p)$ factors as a product $p \prod_{i=1}^{n}\left(p-c_{i}\right)$, where the numbers $c_{i} \in \mathbb{N}$ can be read off from the graph $G$. The authors are not aware of an expression for the characteristic polynomial of $\operatorname{Shi}(G)$ for general graphs $G$ in previous literature.

Corollary 3.2. Let $G \subset\left(\begin{array}{c}{[n]} \\ 2\end{array}\right)$ be a graph on $n$ vertices. The arrangements $\operatorname{Shi}(G)$ and $\operatorname{Ish}(G)$ have the same number of regions.

Proof. Combine Theorem 3.1 and Zaslavsky's Theorem [14].

No bijective proof of Corollary 3.2 is known.

\section{Labelling the Regions}

In this section we will describe how to label the regions of the Shi and Ish arrangements $\operatorname{Shi}(G)$ and Ish $(G)$. These labels will be called Shi ceiling diagrams and Ish ceiling diagrams and will be designed to keep track of the ceilings and degrees of freedom of these regions. (Something like "Shi floor diagrams" appeared earlier in the work of Athanasiadis and Linusson [2].)

\subsection{Shi ceiling diagrams}

We denote by $C$ the dominant cone in the Coxeter arrangement $\operatorname{Cox}(n)$ defined by the coordinate inequalities $x_{1}>x_{2}>\ldots>x_{n}$. The action of the symmetric group $\mathfrak{S}(n)$ on $\mathbb{R}^{n}$ by coordinate permutation induces a simply transitive action of $\mathfrak{S}(n)$ on the regions of $\operatorname{Cox}(n)$, so that every region of $\operatorname{Cox}(n)$ can be uniquely written as $w C$ for some $w \in \mathfrak{S}(n)$.

Let $G \subseteq\left(\begin{array}{c}{[n]} \\ 2\end{array}\right)$ be a fixed graph on $n$ vertices. For $w \in \mathfrak{S}(n)$, we define a poset $\Phi^{+}(G, w)$ (i) as follows. As a set, $\Phi^{+}(G, w)$ consists of the following affine hyperplanes in $\operatorname{Shi}(G)$ :

$$
\Phi^{+}(G, w):=\left\{x_{w(i)}-x_{w(j)}=1: i<j, w(i)<w(j), w(i) w(j) \in G\right\} .
$$

On can see that the hyperplanes in $\Phi^{+}(G, w)$ are precisely the hyperplanes in $\operatorname{Shi}(G)$ which intersect the cone $w C$. The partial order on $\Phi^{+}(G)$ is defined by

$$
\left(x_{w\left(i^{\prime}\right)}-x_{w\left(j^{\prime}\right)}=1\right) \preceq\left(x_{w(i)}-x_{w(j)}=1\right)
$$

if

$$
w(i) \leq w\left(i^{\prime}\right)<w\left(j^{\prime}\right) \leq w(j) .
$$

Given $w \in \mathfrak{S}(n)$, we will label the regions of $\operatorname{Shi}(G)$ contained in $w C$ with order ideals in the poset $\Phi^{+}(G, w)$.

Theorem 4.1. There is a bijection between regions of $\operatorname{Shi}(G)$ which are contained in the cone $w C$ and order ideals (down-closed sets) in the poset $\Phi^{+}(G, w)$. This bijection is given by sending a region $R$ contained in $w C$ to the set of hyperplanes in $\Phi^{+}(G, w)$ which do not separate $R$ from the origin. The maximal elements of this ideal are the ceilings of $R$.

(i) The notation $\Phi^{+}(G, w)$ is due to the fact that when $G=K_{n}$ is the complete graph and $w \in \mathfrak{S}(n)$ is the identity permutation, the poset $\Phi^{+}(G, w)$ is isomorphic to the type $\mathrm{A}_{n-1}$ positive root poset. 

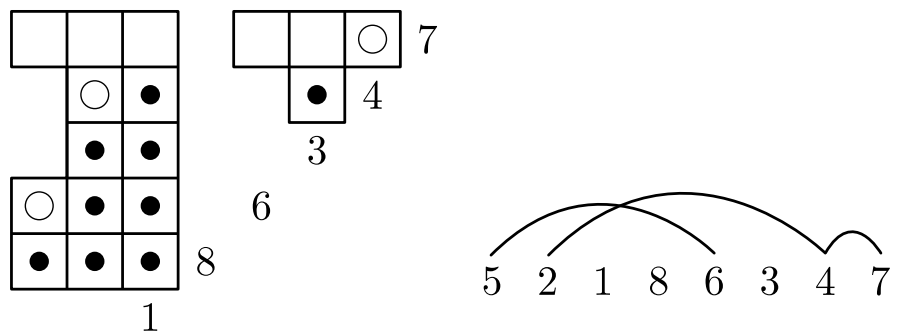

2

5

Fig. 4: An order ideal (left) and a Shi ceiling diagram (right)

Proof. Omitted.

For example, let $n=8, G=K_{8}$, and $w=51286347$. The poset $\Phi^{+}(G, w)$ is shown on the left of Figure 4, with partial order increasing up and to the left. The elements of this poset which contain circles form an order ideal in this poset. The hollow circles are the maximal elements of this order ideal. The ceilings of the corresponding region of Shi(8) are $x_{5}-x_{8}=1, x_{1}-x_{6}=1$, and $x_{3}-x_{7}=1$.

Given a region $R$ of $\operatorname{Shi}(G)$, define the Shi ceiling diagram of $R$ as follows. Let $w \in \mathfrak{S}(n)$ be the unique permutation such that $R \subseteq w C$. Write the one-line notation $w(1) w(2) \ldots w(n)$ of $w$ on a line. For every maximal element $x_{w(i)}-x_{w(j)}=1$ of the order ideal in $\Phi^{+}(G, w)$ corresponding to $R$ as in Theorem 4.1, draw an arc connecting $w(i)$ and $w(j)$. Theorem 4.1 guarantees that the partition $\pi$ of $[n]$ generated by $i \sim j$ if $w(i)$ and $w(j)$ are connected by an arc is nonnesting. The pair $(w, \pi)$ is the Shi ceiling diagram of $[n]$. The Shi ceiling diagram of the region of $\operatorname{Shi}(8)$ from the previous paragraph is shown on the right of Figure 4 The corresponding pair $(w, \pi)$ is $(51286347,\{\{1,4\},\{2,5\},\{3\},\{6,8\},\{7\}\})$. Figure 5 shows the arrangement $\operatorname{Shi}(3)$ with its regions labeled by their Shi ceiling diagrams.

Shi ceiling diagrams label the regions of $\operatorname{Shi}(G)$. They also can be used to read of the degrees of freedom of a region.

Lemma 4.2. The set of Shi ceiling diagrams of the regions of $\operatorname{Shi}(G)$ is exactly the set of pairs $(w, \pi)$ where $w \in \mathfrak{S}(n)$ and $\pi$ is a nonnesting partition of $[n]$ satisfying:

- if $i<j$ is an arc in the arc diagram of $\pi$, then $w(i)<w(j)$,

- if $i<j$ is an arc in the arc diagram of $\pi$, then $w(i) w(j) \in G$.

Moreover, if $(w, \pi)$ is a pair of a permutation $w \in \mathfrak{S}(n)$ and a nonnesting partition $\pi$ of $[n]$ satisfying the two conditions above, then there exists a unique region $R$ of $\operatorname{Shi}(G)$ with Shi ceiling diagram $(w, \pi)$. The number of ceilings of $R$ is the number of arcs in the arc diagram of $\pi$. The number of degrees of freedom of $R$ is the number of connected components of $\pi$.

Proof. Omitted.

For example, the region of $\operatorname{Shi}(8)$ whose Shi ceiling diagram is shown in Figure 4 has two degrees of freedom. 


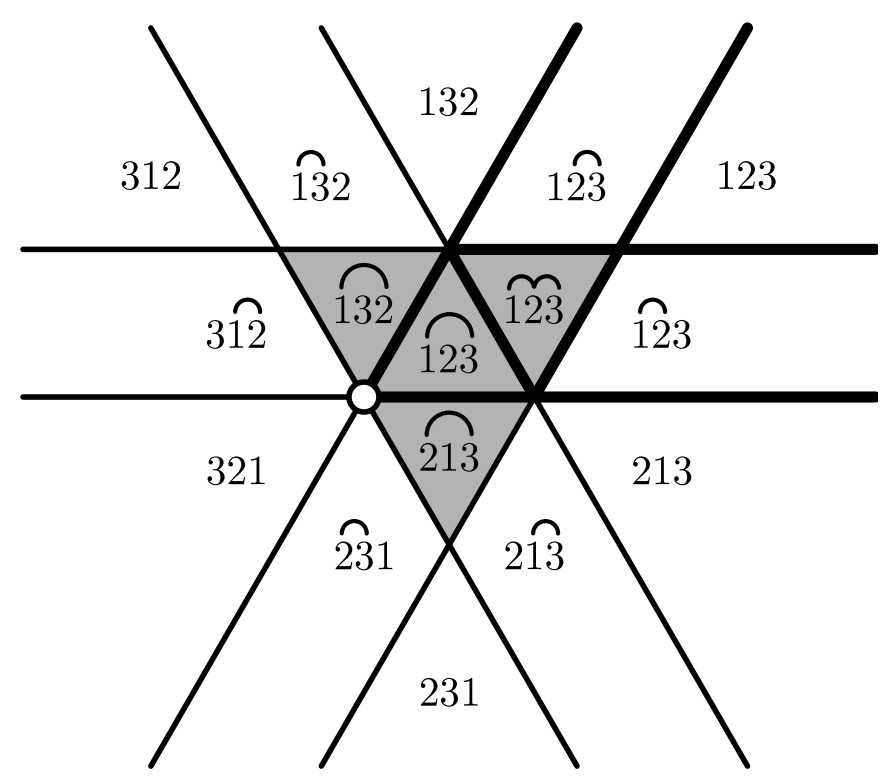

Fig. 5: The regions of Shi(3) and their Shi ceiling diagrams.

\subsection{Ish ceiling diagrams}

In order to compare the Shi and Ish arrangements, we will introduce an Ish-analog of the Shi ceiling diagrams. Fix a graph $G \subseteq\left(\begin{array}{c}{[n]} \\ 2\end{array}\right)$.

Given a permutation $w \in \mathfrak{S}(n)$, we define a poset $\Psi^{+}(G, w)$ as follows. As a set, $\Psi^{+}(G, w)$ is the following collection of affine hyperplanes in $\operatorname{Ish}(G)$ :

$$
\Psi^{+}(G, w):=\left\{x_{1}-x_{j}=i: i<j \in G, w^{-1}(i)<w^{-1}(j)\right\} .
$$

It can be shown that $\Psi^{+}(G, w)$ is exactly the set of hyperplanes in $\operatorname{Ish}(G)$ which intersect the region $w C$ of $\operatorname{Cox}(n)$. The partial order on $\Psi^{+}(G, w)$ is generated by

$$
\left(x_{1}-x_{j}=i\right) \prec\left(x_{1}-x_{j^{\prime}}=i^{\prime}\right)
$$

if $j<j^{\prime}$ or $i<i^{\prime}$. In analogy with the case of the Shi arrangement, regions of $\operatorname{Ish}(G)$ which are contained in the cone $w C$ are in a natural bijection with order filters in $\Psi^{+}(G, w)$.

Theorem 4.3. There is a bijection between regions of $\operatorname{Ish}(G)$ which are contained in the cone $w C$ and order filters (up-closed sets) in the poset $\Psi^{+}(G, w)$. This bijection is given by sending a region $R$ to the set of hyperplanes in $\Psi^{+}(G, w)$ which do not separate $R$ from the origin. The minimal elements of the ideal corresponding to $R$ are the ceilings of $R$.

Proof. Omitted.

It is convenient to express Theorem 4.3 with a picture. Given $w \in \mathfrak{S}(n)$, we draw $w(1), w(2), \ldots, w(n)$ on a line. For each $j$ to the right of 1 , we draw $j-1$ boxes above the symbol $j$. If we identify the $i$ th 


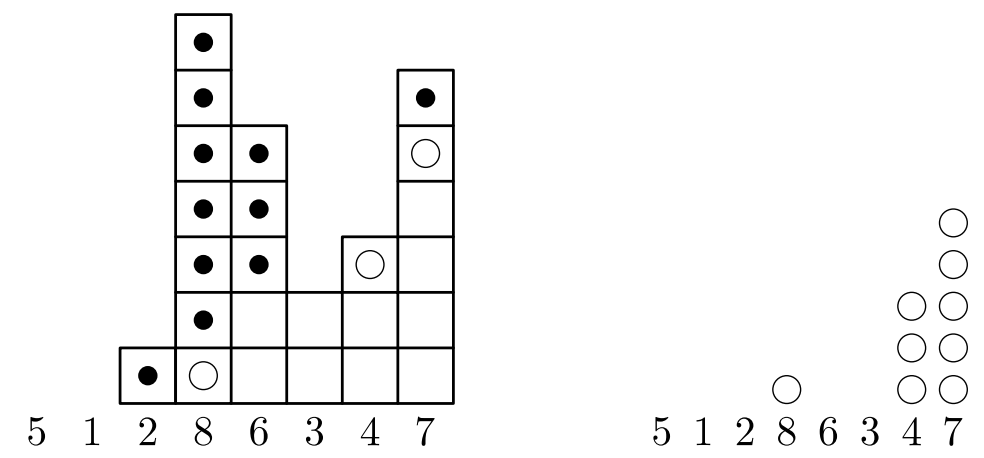

Fig. 6: An order filter (left) and an Ish ceiling diagram (right)

box above $j$ with the hyperplane $x_{1}-x_{j}=i$ and erase the boxes which correspond to hyperplanes not in Ish $(G)$, we obtain the poset $\Psi^{+}(G, w)$; the partial order increases up and to the left. Given a region $R$ of Ish $(G)$ contained in $w C$, we can draw its order filter in $\Psi^{+}(G, w)$ using this pictorial representation.

For example, let $n=8, G=K_{8}$, and $w=51286347$. Figure 6 shows the poset $\Psi^{+}(G, w)$, with partial order increasing up and to the left. The boxes in this poset which contain circles form an order filter; the minimal elements in this order filter are the hollow circles. The ceilings of the corresponding region $R$ are $x_{1}-x_{8}=1, x_{1}-x_{4}=3$, and $x_{1}-x_{7}=5$.

The pictorial representation of order filters in the posets $\Psi^{+}(G, w)$ can be used to define Ish-analogs of Shi ceiling diagrams. Given a region $R$ of $\operatorname{Ish}(G)$, the Ish ceiling diagram of $R$ is the pair $(w, \epsilon)$ defined as follows. We let $w \in \mathfrak{S}(n)$ be the unique permutation such that $R$ is contained in the region $w C$ of $\operatorname{Cox}(n)$. We draw the poset $\Psi^{+}(G, w)$ and the order filter corresponding to $R$ as in the previous paragraph. We let $\epsilon=\epsilon_{1} \ldots \epsilon_{n}$ be the sequence of nonnegative integers defined by setting $\epsilon_{j}=0$ if there is no minimal element of the order filter of $R$ above $w(j)$ in this representation and by setting $\epsilon_{j}=i$ if there is a minimal element in the order filter of $R$ above $w(j)$ and this minimal element is $x_{1}-x_{w(j)}=i$. We $(w, \epsilon)$ visually by drawing $w(1), \ldots, w(n)$ on a line and placing $\epsilon_{i}$ circles above $w(i)$ for all $i$.

The right of Figure 6 shows the Ish ceiling diagram corresponding to the order ideal on the left of Figure 6. The corresponding pair $(w, \epsilon)$ is $(51286347,00010035)$. Figure 7 shows the regions of Ish $(3)$ labeled with their Ish ceiling diagrams.

The Ish ceiling diagrams label the regions of $\operatorname{Ish}(G)$ and can be used to read off the degrees of freedom of a region.

Lemma 4.4. The set of Ish ceiling diagrams of the regions of $\operatorname{Ish}(G)$ is exactly the set of pairs $(w, \epsilon)$ where $w \in \mathfrak{S}(n)$ and $\epsilon=\epsilon_{1} \ldots \epsilon_{n}$ is a sequence of nonnegative integers satisfying:

- $\epsilon_{i}<w(i)$ for all $i$,

- if $\epsilon_{i}>0$, then $w^{-1}(1)<i$,

- if $\epsilon_{i}>0$, then $\epsilon_{i}<w(i) \in G$, and

- if $i<j$ and $\epsilon_{i}, \epsilon_{j}>0$, then $\epsilon_{i}<\epsilon_{j}$. 


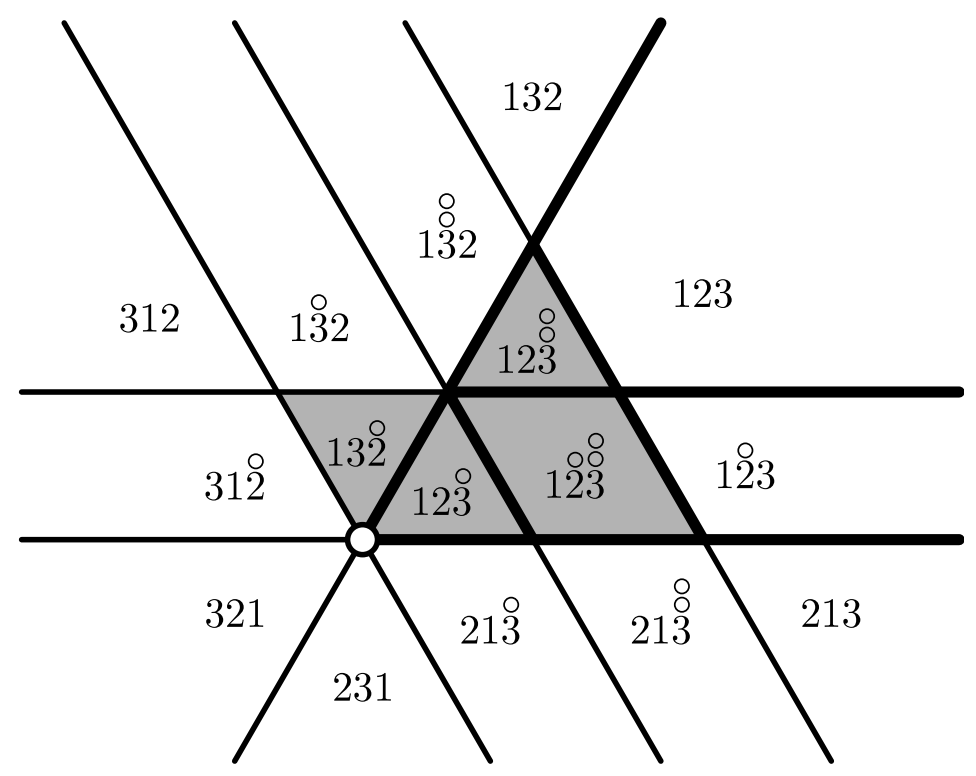

Fig. 7: The arrangement Ish(3) labeled with Ish ceiling diagrams

Given a pair $(w, \epsilon)$ satisfying the four properties listed above, there exists a unique region $R$ of $\operatorname{Ish}(G)$ with Ish ceiling diagram $(w, \epsilon)$. The number of ceilings of $R$ is the number of positive entries of $\epsilon$. The number of degrees of freedom of $R$ is $n+w^{-1}(1)-j$, where $j=\max \left\{i: \epsilon_{i}>0\right\}$ if $\epsilon$ is not the zero sequence and $j=w^{-1}(1)$ if $\epsilon$ is the zero sequence.

Proof. Omitted.

For example, the region $R$ of Ish(8) whose Ish ceiling diagram is shown in Figure 6 has $8+2-8=2$ degrees of freedom.

\section{Counting the regions}

In this section we use Shi and Ish ceiling diagrams to obtain our equidistribution results regarding the regions of $\operatorname{Shi}(G)$ and $\operatorname{Ish}(G)$. To do this, we will introduce a new set partition-valued statistic on the regions of $\operatorname{Shi}(G)$ and $\operatorname{Ish}(G)$ called "ceiling partition". Two regions with the same ceiling partition will also have the same number of ceilings. We will prove that the bistatistic (ceiling partition, degrees of freedom) has the same joint distribution on the regions of $\operatorname{Shi}(G)$ and $\operatorname{Ish}(G)$ and compute this joint distribution explicitly. This will imply that the joint distribution of (number of ceilings, degrees of freedom) is the same on the regions of $\operatorname{Shi}(G)$ and $\operatorname{Ish}(G)$. We will also give an explicit bijection between the dominant regions of $\operatorname{Shi}(G)$ and $\operatorname{Ish}(G)$ which preserves ceiling partitions (thus preserving number of ceilings).

Definition 5.1. 1. Let $R$ be a region of $\operatorname{Shi}(G)$. The ceiling partition of $R$ is the set partition $\tau$ of $[n]$ generated by $i \sim j$ if $x_{i}-x_{j}=1$ is a hyperplane in $\operatorname{Shi}(G)$ and a ceiling of $R$. 
2. Let $R$ be a region of $\operatorname{Ish}(G)$. The ceiling partition of $R$ is the set partition $\tau$ of $[n]$ generated by $i \sim j$ if $x_{1}-x_{j}=i$ is a hyperplane in $\operatorname{Ish}(G)$ and a ceiling of $R$.

The type of a region $R$ of $\operatorname{Shi}(G)$ or $\operatorname{Ish}(G)$ is a $G$-deleted partition of $[n]$. We remark that the ceiling partition of a region of $\operatorname{Shi}(G)$ or $\operatorname{Ish}(G)$ is neither noncrossing nor nonnesting in general.

Lemma 5.1. Let $G \subseteq\left(\begin{array}{c}{[n]} \\ 2\end{array}\right)$ be a graph on $n$ vertices, let $R$ be a region of $\operatorname{Shi}(G)$ or of $\operatorname{Ish}(G)$, and let $\tau$ be the ceiling partition of $R$. If $\tau$ has $k$ blocks, then $R$ has $n-k$ ceilings.

Proof. Omitted.

Theorem 5.2. Let $G \subseteq\left(\begin{array}{c}{[n]} \\ 2\end{array}\right)$ be a graph on $n$ vertices and let $\tau$ be a G-deleted partition of $[n]$. If $\tau$ is not nonnesting, there are no dominant regions of $\operatorname{Shi}(G)$ or of $\operatorname{Ish}(G)$ with ceiling partition $\tau$. If $\tau$ is nonnesting, there exists a unique dominant region of $\operatorname{Shi}(G)$ with ceiling partition $\tau$ and a unique dominant region of $\operatorname{Ish}(G)$ with ceiling partition $\tau$.

Proof. This is essentially a picture proof. For the identity permutation $w=\mathbf{1}$ we observe that the posets $\Phi^{+}(G, \mathbf{1})$ and $\Psi^{+}(G, \mathbf{1})$ look exactly the same, except that one is reflected in a line of slope 1 . For example, here are the posets corresponding to the graph $G=\left(\begin{array}{c}{[8]} \\ 2\end{array}\right)-\{14,34,48,58\}$; Shi on the left, Ish on the right:
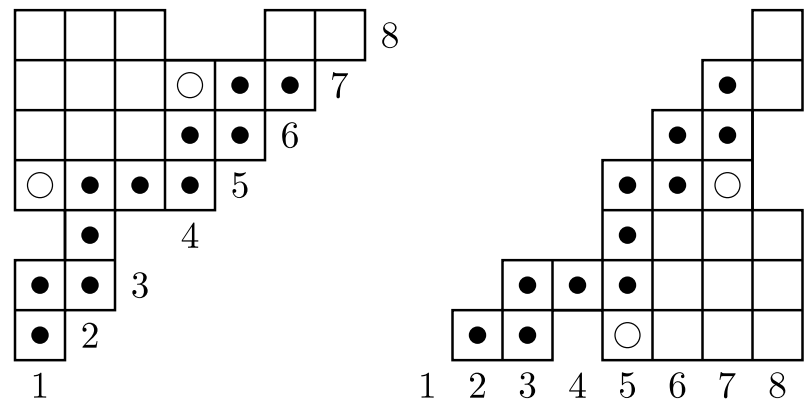

This reflection is an order-reversing bijection between $\Phi^{+}(G, \mathbf{1})$ and $\Psi^{+}(G, \mathbf{1})$. Hence it induces a bijection between ideals in $\Phi^{+}(G, \mathbf{1})$ with $c$ maximal elements (dominant $\operatorname{Shi}(G)$-regions with $c$ ceilings) and filters in $\Psi^{+}(G, \mathbf{1})$ with $c$ minimal elements (dominant $\operatorname{Ish}(G)$-regions with $c$ ceilings). This bijection preserves ceiling partitions.

The bijection given in Theorem 5.2 does not extend to regions outside the dominant cone because the posets $\Phi^{+}(G, w)$ and $\Psi^{+}(G, w)$ look very different in general for permutations $w$ other than 1 . This bijection does not preserve degrees of freedom - indeed, it cannot as a glance at Figure 1 shows that for $n=3$ and $G=K_{3}$ the arrangement $\operatorname{Shi}(3)$ has two dominant regions with one degree of freedom while the arrangement Ish(3) has three dominant regions with one degree of freedom.

Theorem 5.3. Let $G \subseteq\left(\begin{array}{c}{[n]} \\ 2\end{array}\right)$ be a graph on $n$ vertices, let $1 \leq d \leq n$, and let $\tau$ be a $G$-deleted partition of $[n]$ with $k$ blocks.

1. The number of regions of $\operatorname{Shi}(G)$ or of $\operatorname{Ish}(G)$ with ceiling partition $\tau$ is

$$
\frac{n !}{(n-k+1) !} \text {. }
$$


2. The number of regions of $\operatorname{Shi}(G)$ or of $\operatorname{Ish}(G)$ with ceiling partition $\tau$ and with d degrees of freedom is

$$
\frac{d(n-d-1) !(k-1) !}{(n-k-1) !(k-d) !} .
$$

Proof. (Sketch.) Use Lemmas 4.2 and 4.4 to count Shi and Ish ceiling diagrams which label regions with the desired properties. In the case of the Ish arrangement, both parts are routine counting arguments. In the case of the Shi arrangement, Part 1 requires a product formula due to Kreweras [7] which counts nonnesting partitions of $[n]$ with fixed type and Part 2 requires a product formula due to the second author [10] which counts nonnesting partitions of $[n]$ with fixed type and a fixed number of connected components.

\section{References}

[1] D. Armstrong. Hyperplane arrangements and diagonal harmonics. In preparation, 2010.

[2] C. Athanasiadis and S. Linusson. A simple bijection for the regions of the Shi arrangement of hyperplanes. Discrete Math., 204 (1999) pp. 27-39.

[3] C. A. Athanasiadis. Characteristic polynomials of subspace arrangements and finite fields. $A d v$. Math., 122 (1996) pp. 193-233.

[4] C. A. Athanasiadis. On free deformations of the braid arrangement. European J. Combin., 19 (1997) pp. 7-18.

[5] H. Crapo and G.-C. Rota. On the Foundations of Combinatorial Theory: Combinatorial Geometries. MIT Press, Cambridge, MA (1970). Preliminary edition.

[6] P. Headley. On a family of hyperplane arrangements related to affine Weyl groups. J. Alg. Combin., 6, 4 (1997) pp. 331-338.

[7] G. Kreweras. Sur les partitions non croisées d'un cycle. Discrete Math., 1, 4 (1972) pp. 333-350.

[8] P. Orlik and K. Terao. Arrangements of Hyperplanes. Springer-Verlag, New York, NY (1992).

[9] A. Postnikov and R. Stanley. Deformations of Coxeter hyperplane arrangements. J. Combin. Theory Ser. A, 91, (2000) pp. 544-597.

[10] B. Rhoades. Enumeration of connected Catalan objects by type. Accepted, European J. Combin., 2010. Available on arXiv: 1005.2553.

[11] J.-Y. Shi. The Kazhdan-Lusztig cells in certain affine Weyl groups. Lecture Notes in Mathematics, no. 1179, Springer-Verlag, Berlin/Heidelberg/New York (1986).

[12] R. Stanley, Enumerative Combinatorics, vol. 1, Cambridge University Press, Cambridge, (1997).

[13] M. Wachs and J. Walker. On geometric semilattices. Order, 2, 4, (1986), pp. 367-385.

[14] T. Zaslavsky. Facing up to arrangements: a face-count for partitions of space by hyperplanes. Mem. Amer. Math. Soc., 1 (1975). 